\title{
Transformación de fundación en cooperativa
}

\author{
Juan Ramón Manzano Malaxechevarría \\ Notario del Ilustre Colegio Notarial del País Vasco
}

Sumario: I. Introducción: a) La Ley de Cooperativas de Euskadi. b) El Reglamento de la Ley de Cooperativas de Euskadi. c) El concepto de fundación. d) El concepto de cooperativa. e) Clases de cooperativas. f) Clases de socios en las cooperativas. g) La baja de los socios en las cooperativas y el derecho de retorno. h) Menciones a la transformación hechas en la escritura de transformación de «Fundación Peñascal» en sociedad cooperativa. i) Compromiso. j) La cooperativa de integración social. k) La transformación de fundación en cooperativa desde una perspectiva fiscal. I) La sociedad cooperativa pequeña de la Ley 6/2008. II. Notas bibliográficas.

Resumen: El autor dibuja el camino entre una entidad de interés general, como es una fundación, hacia otra que, sin ser una entidad de interés general, no es tampoco una persona jurídica de carácter mercantil puro, como es una cooperativa. La transformación de fundación en cooperativa exige una interpretación amplia del principio de autonomía de la voluntad que el legislador favorece.

Palabras clave: Economía social, fundaciones, cooperativas, transformación mercantil.

Abstract: The author charts a path between entities that meet needs in the general interest such as foundations and others which are neither entities of general interest nor have purely mercantile legal personality such as cooperatives. Transforming a foundation into a cooperative calls for a broad interpretation of the principle of free will that the legislation wishes to foster.

Key words: Social economics, foundations, cooperatives, mercantile transformation. 


\section{Introducción}

Aunque el asunto que hemos de tratar hoy es de carácter más bien técnico, no olvidemos que se refiere a entidades que se dedican a la integración social de personas desfavorecidas y que, según datos publicados por «El Correo» del sábado 1 de diciembre de 2012, un 5,7\% de la población en Euskadi no cubre sus gastos básicos, un 24,5\% padece dificultades para afrontar sus gastos habituales, un $12,3 \%$ ha de recurrir en cuantía significativa a sus ahorros para financiar esos gastos, un 4,7\% sufre problemas graves de alimentación, un 5,9\% incurre en impagos o atrasos y un 8,3\% padece de frío en su casa. Se escucha al fondo un rumor de desahucios, de carencias y de buenas intenciones, pero no parece que acertemos a encontrar el camino para salir de una situación tan triste. En estas circunstancias, los esfuerzos para ayudar a quienes atraviesan momentos tan difíciles no pueden suscitar más que admiración y solidaridad. Y los intentos para encontrar las formas jurídicas más adecuadas para conseguir fines tan justos y tan útiles no son una simple filigrana para ocupación de los expertos, sino actividades de primera y urgente necesidad social. En nuestra actividad profesional tenemos a veces la suerte de haber sido testigos de esos esfuerzos y de esos intentos, y de saber que las personas que se han implicado en ellos han actuado con generosidad y dedicación y que su tarea es útil y es necesaria.

Detrás de cuanto hoy diremos está la economía social, una pequeña isla de autorregulación en una economía globalizada, en la que el Estado no alcanza a resolverlo todo, por no decir que se ve obligado a resolver tan sólo lo que no se resuelve en un ámbito mayor. Pero, aunque la isla sea pequeña, creamos en la posibilidad de una isla, de muchas islas, en la de un archipiélago en crecimiento de esta forma particular de empresa, que fue ya considerada como tal en el Código de Comercio. Pues si la empresa es en un sentido económico la «organización de los factores de la producción (capital y trabajo) para la obtención de un lucro ilimitado» y en un sentido jurídico-mercantil «el ejercicio profesional de una actividad económica planificada con la finalidad de intermediar en el mercado de bienes y servicios», admite distintas configuraciones. Y la creciente intervención pública y planificación de la economía permite considerar empresas no sólo a las que pretenden obtener un lucro, sino también a las que persiguen fines cien-

Joaquin Garrigues, Curso de Derecho Mercantil, I, p. 159.

2 Rodrigo Uría, Derecho Mercantil, Marcial Pons, Madrid, 1989, p. 37. 
tíficos, sociales o incluso benéficos que se organizan como tales con el sólo propósito de producir bienes y servicios y que utilizan para ello las formas de autoorganización propias del cooperativismo.

El Estado no ha sido ajeno a la realidad de la empresa cooperativa y ya reguló las cooperativas en la Ley de 2 de enero de 1942 y en su Reglamento de 11 de noviembre de 1943, así como en el Código de Comercio, que en su artículo 124 dispuso que "... las cooperativas de producción, de crédito o de consumo, sólo se considerarán mercantiles y quedarán sujetas a las disposiciones de este Código cuando se dediquen a actos de comercio extraños a la mutualidad o se convirtieren en sociedades a prima fija.» Y no sólo eso, sino que ha puesto ese tipo de empresa en un lugar preeminente del ordenamiento jurídico, en el Título VII de la Constitución Española (CE), relativo a Economía y Hacienda, en el apartado segundo del artículo 129 se dice así: "Los poderes públicos promoverán eficazmente las diversas formas de participación en la empresa y fomentarán, mediante una legislación adecuada las sociedades cooperativas. También establecerán los medios que faciliten el acceso de los trabajadores a la propiedad de los medios de producción.»

Y como la materia cooperativa no se cuenta entre las reservadas al Estado por el artículo 149.1 CE ni entre las que el artículo 148.1 considera como asumibles por las Comunidades Autónomas, éstas acudieron a la vía del art. 149.3 CE («Las materias no atribuidas expresamente al Estado por la Constitución podrán corresponder a las CCAA en virtud de sus respectivos estatutos») para poder regularlas. Y así, la primera Ley autonómica aprobada fue la Ley Vasca de Cooperativas (Ley 1/1982, de 11 de febrero), a la que siguió luego la Ley 4/1993, de 24 de junio, de Cooperativas de Euskadi (LCE), que es objeto de mi intervención de hoy, en la que voy a tratar de la transformación de fundación en sociedad cooperativa, un viaje para cuyo análisis hemos de partir de una definición, siquiera sea sumaria y no muy rigurosa del punto de partida y el de llegada.

Veamos, pues, para empezar, de dónde a dónde vamos. O lo que es lo mismo, qué es una fundación y qué es una cooperativa, pues eso nos indicará de entrada si nuestro propósito en alcanzable.

El punto de partida es una fundación. Y fundación es una organización constituida sin ánimo de lucro que por voluntad de sus creadores tiene afectado de modo duradero su patrimonio a la realización de fines de interés general, tales como la asistencia social, cívicos, educativos, deportivos, científicos, culturales, sanitarios, de cooperación para el desarrollo, de defensa del medio ambiente u otros de naturaleza análoga. 
Y el punto de llegada es una cooperativa, es decir, por utilizar una definición legal, «... aquella sociedad que desarrolla una empresa que tiene por objeto prioritario la promoción de las actividades económicas y sociales de sus miembros y la satisfacción de sus necesidades con la participación activa de los mismos, observando los principios del cooperativismo y atendiendo a la comunidad de su entorno» (artículo 1 de la Ley 4/1993, de Cooperativas de Euskadi).

Se trata, pues, de hacer el camino entre una entidad de interés general y otra que no lo es, si bien esta segunda no es tampoco una persona jurídica de carácter mercantil puro, o sea, dirigida a la obtención de un lucro privado partible entre los socios, sino más bien destinada a la satisfacción sin ánimo de lucro de necesidades de éstos de un modo regido por los principios del cooperativismo, que no dejan de estar impregnados de un cierto carácter social, o sea, de autoorganización y de limitación de la ganancia a la satisfacción de un interés de los socios y no de obtención de una ganancia ilimitada y acumulable, más allá de la satisfacción de todo interés concreto. Y este camino, si no difícil de admitir conceptualmente, exige al menos una interpretación amplia del principio de autonomía de la voluntad, interpretación que el legislador ha favorecido de la manera que a continuación vamos a considerar.

\section{a) La Ley de Cooperativas de Euskadi}

En la Ley 4/1993, de Cooperativas de Euskadi, modificada por la Ley $1 / 2000$, de 29 de junio, al regular las modificaciones estatutarias en esta clase de entidades, indica cuáles son los requisitos que habrá de reunir la modificación de los estatutos de la fundación para transformarlos en los de una Cooperativa con respeto a lo establecido en su Ley rectora, es decir, con un capital social mínimo de tres mil euros (art. 4 LCE), aportado en moneda de curso legal, salvo que la Asamblea General o los Estatutos acuerden otra cosa (art. 57.3 LCE), con tres socios como mínimo (art. 19.1 LCE) y con cumplimiento de las exigencias que la propia Ley impone a fin de inscribir la constitución en el Registro de Cooperativas, inscripción que tiene carácter constitutivo y sin la cual no hay cooperativa posible.

Ello supone que la modificación de los estatutos de la fundación, que habrá de ser acordada por el órgano de gobierno (art. 31 LFPV), cumpla dos condiciones: que se respete el fin fundacional y que no lo prohíba la persona fundadora. Se presumirá que la modificación de estatutos aprobada por el órgano de gobierno respeta el fin fundacional y no será necesaria la aprobación del protectorado, salvo que la modifi- 
cación consista en cambio de los fines de la fundación. El respeto al fin fundacional es, por tanto, el límite conceptual de esta clase de operaciones, pues si el fin de la cooperativa que se crea fuese diferente del de la fundación la transformación no es posible. Es decir, debe haber una identidad de fin entre la fundación previamente existente y la cooperativa que de la transformación de ella ha de nacer.

El paraguas protector de tal transformación lo brinda intencionadamente la propia Ley en su artículo 86, que dice así:

\section{Artículo 86. Transformación en cooperativas}

1. Las sociedades y las agrupaciones de carácter no cooperativo podrán transformarse en cooperativas de alguna de las clases reguladas en la presente Ley, siempre que no exista precepto legal que lo prohíba expresamente.

2. La transformación será acordada por la Junta General o mediante el sistema válido equivalente para expresar la voluntad social, con la mayoría exigida por la legislación aplicable, no afectará a la personalidad jurídica de la entidad transformada y se hará constar en escritura pública, que expresará necesariamente todas las menciones previstas en esta Ley para la constitución de una cooperativa.

3. La escritura pública de transformación, a la que se incorporará, en su caso, el informe de los expertos independientes sobre el patrimonio social no dinerario, se presentará para su inscripción en el Registro Mercantil y demás procedentes, en su caso, y siempre en el de cooperativas, acompañada del balance cerrado el día anterior al del acuerdo de transformación.

4. La transformación en cooperativa no altera el anterior régimen de responsabilidad de los socios de la entidad transformada por las deudas sociales contraídas con anterioridad a la transformación de la entidad, a no ser que los acreedores hayan consentido expresamente la transformación.

Pero, sobre todo, la admisibilidad de la transformación debemos buscarla en el principio de autonomía de la voluntad, consagrado en el artículo 1.255 del Código Civil y recogido en el artículo 12.3 de la Ley de Cooperativas de Euskadi, según el cual «... en la escritura de constitución podrán incluirse además todos los pactos y condiciones que los promotores hubiesen acordado en la Asamblea Constituyente, siempre que no se opongan a las leyes ni contradigan los principios configuradores de la sociedad cooperativa». Y fue fundándose en este principio como el Consejo Superior de Cooperativas de Euskadi (CSCE) autorizó la transformación de la «Fundación Peñascal» en «Peñascal, S. Coop., Cooperativa de Trabajo Asociado», sin ánimo de lucro, de iniciativa so- 
cial, que se rige por las disposiciones de la Ley 4/1993, de Cooperativas de Euskadi, modificada por la Ley 1/2000, de 29 de junio, por la Ley 8/2006, de 1 de diciembre, y por la disposición adicional cuarta de la Ley 6/2008, de 25 de junio, todas ellas aprobadas por el Parlamento Vasco, así como por lo dispuesto en el Decreto 61/2000, de 4 de abril, que regula las cooperativas de iniciativa social.

Dicha autorización implica que el CSCE se reserva el control de los fondos consignados en el citado Fondo de Reserva y, a fin de garantizar su conservación y aplicación a los fines de la cooperativa, el derecho de autorizar la modificación de sus estatutos si así fuera acordada por la Asamblea General.

\section{b) El Reglamento de la Ley de Cooperativas de Euskadi}

Y en el Decreto 59/2005, de 29 de marzo, por el que se aprueba el Reglamento de organización y funcionamiento del Registro de Cooperativas de Euskadi, se regula esta transformación expresamente en su artículo 60, que dice así:

\section{Transformación en cooperativa}

Para su inscripción en el Registro regulado en el presente Reglamento, la escritura pública de transformación en cooperativa de otro tipo de Sociedad o Agrupación de carácter no cooperativo, deberá contener las menciones a que se refiere el número 2 del artículo 86 de la Ley de Cooperativas de Euskadi. Dicha escritura irá acompañada del balance previsto en el número 3 del mismo precepto y, en su caso, incorporará el informe allí mencionado, y cuando la Sociedad transformada sea de Responsabilidad Limitada se incorporará, además, el balance final cerrado el día anterior al otorgamiento de la escritura.

2. Una vez inscrita la transformación en Cooperativa en el Registro de Cooperativas de Euskadi, se comunicará, en su caso, al Registro en que conste inscrita con anterioridad la Sociedad transformada, a los efectos registrales oportunos.

\section{c) El concepto de fundación}

El derecho de fundación viene reconocido en el artículo 34 Constitución Española. Las fundaciones están reguladas en el ámbito estatal por la Ley 50/2002, de 26 de diciembre, que las define como organizaciones constituidas sin ánimo de lucro que por voluntad de sus crea- 
dores tienen afectado de modo duradero su patrimonio a la realización de fines de interés general (artículo 2), tales como la asistencia social, cívicos, educativos, deportivos, científicos, culturales, sanitarios, de cooperación para el desarrollo, de defensa del medio ambiente u otros de naturaleza análoga. Las fundaciones se rigen por la voluntad del fundador, por sus Estatutos y por la citada ley. Pueden constituir fundaciones tanto las personas físicas (p. ej., la Fundación Josep Carreras contra la leucemia) como las jurídicas (p. ej., la Fundación Real Madrid); pueden constituirse por acto inter vivos mediante escritura pública o por acto mortis causa en testamento. Las fundaciones adquieren personalidad jurídica desde su inscripción en el correspondiente Registro de Fundaciones. Y en el País Vasco la legislación principal está constituida por la Ley 12/1994, de 17 de junio, de Fundaciones del País Vasco, el Decreto 101/2007, de 19 de junio, por el que se aprueba el Reglamento del Registro de Fundaciones del País Vasco y el Decreto 404/1994, de 18 de octubre, por el que se aprueba el Reglamento de organización y funcionamiento del Protectorado y del Registro de Fundaciones del País Vasco.

Las fundaciones tienen tres elementos esenciales: el fin, el patrimonio y la organización.

El fin de una fundación es el elemento identificador de la misma y debe ser de interés general.

La modificación de los estatutos de la fundación podrá ser acordada por el órgano de gobierno (art. 31 LFPV), siempre que se cumplan dos condiciones: que se respete el fin fundacional y que no lo prohíba la persona fundadora. Se presumirá que la modificación de estatutos aprobada por el órgano de gobierno respeta el fin fundacional y no será necesaria la aprobación del protectorado, salvo que la modificación consista en cambio de los fines de la fundación.

El órgano de gobierno tiene el deber de acordar la modificación de los estatutos cuando las circunstancias que motivaron la constitución de la fundación hayan variado de tal manera que ésta no pueda actuar satisfactoriamente con arreglo a aquellos, salvo que para el supuesto de que se trate haya previsto el fundador la extinción de la fundación (art. 31.2 LFPV).

\section{d) El concepto de cooperativa}

El concepto legal de cooperativa, en lo que a la exposición de hoy se refiere, es el que recoge el artículo 1 de la Ley 4/1993, de Cooperativas de Euskadi: 
La cooperativa es aquella sociedad que desarrolla una empresa que tiene por objeto prioritario la promoción de las actividades económicas y sociales de sus miembros y la satisfacción de sus necesidades con la participación activa de los mismos, observando los principios del cooperativismo y atendiendo a la comunidad de su entorno (art. 1 Ley 4/1993).

La cooperativa deberá ajustar su estructura y funcionamiento a los principios cooperativos, que serán aplicados en el marco de la Ley. Dentro de ésta, actuará con plena autonomía e independencia respecto de cualesquiera organizaciones y entidades, públicas o privadas.

Las cooperativas pueden realizar cualquier actividad económica o social, salvo expresa incompatibilidad legal basada en la incompatibilidad con las exigencias y principios básicos del cooperativismo.

Como concepto doctrinal, vamos a utilizar el de Carlos Martín GINTO MONZÓN³, en el "Manual de Derecho de Sociedades Cooperativas» publicado por CSCE y el Instituto de Derecho Cooperativo y Economía Social de la Universidad del País Vasco (GEZKI) y coordinado por Santiago MERINO, que es el siguiente: «asociación autónoma de personas que se han unido de forma voluntaria para satisfacer sus necesidades y aspiraciones económicas, sociales y culturales en común mediante una empresa de propiedad conjunta y de gestión democrática».

Según ese mismo autor, los principios cooperativos son los siguientes:

- Adhesión voluntaria y abierta.

- Gestión democrática por parte de los socios.

- Participación económica de los socios.

- Autonomía e independencia.

- Educación, formación e información.

- Cooperación entre cooperativas.

- Interés por la comunidad.

\section{e) Clases de cooperativas}

Son diversos los criterios con arreglo a los cuales pueden clasificarse las cooperativas:

3 Carlos Martín Ginto Monzón, Manual de Derecho de Sociedades Cooperativa, Santiago Merino (coord.), CSCE y GEZKI, Vitoria-Gasteiz, 2007, p. 37. 
a) Atendiendo a su base social:

- De primer grado, en las que pueden ser socios tanto las personas físicas como las jurídicas, aunque la participación de estas últimas está condicionada a lo que establezcan las normas reguladoras de cada clase de cooperativa en función de su objeto social.

- De segundo grado, integradas principalmente por cooperativas, pudiendo también formar parte de ellas socios de trabajo, otras personas jurídicas y empresarios individuales, así como personas físicas, aunque respecto de los socios que no sean cooperativas su participación y número total de votos se reducirá a porcentajes inferiores a los que corresponden a los socios que sean cooperativas.

b) Dentro de las cooperativas de primer grado, y por razón de su objeto, existen las siguientes clases.

- De trabajo asociado.

- De consumidores y usuarios.

- De vivienda.

- Agrarias.

- De explotación comunitaria de tierra.

- De crédito.

- De seguros.

- Sanitarias.

- De servicios empresariales y profesionales.

- De enseñanza.

- De transportes (encuadradas en la Ley dentro de las de servicios profesionales. «Sin perjuicio de lo previsto en la presente Ley sobre cooperativas de trabajo asociado, las personas habilitadas para prestar servicios de transportes de mercancías o de viajeros podrán constituir, al amparo de este artículo cooperativas de transportistas para asumir todas las funciones reconocidas a estas empresas en la legislación sectorial sobre transporte» (art. 124 LCE).

- De integración social.

En la Ley 4/1993, de Cooperativas de Euskadi (LCE), se distinguen las siguientes clases:

- De trabajo asociado.

- De consumo.

- De enseñanza. 
- Agrarias.

- De explotación comunitaria de bienes.

- De vivienda.

- Financieras (de crédito y de seguros).

- Sanitarias.

- De servicios (profesionales, empresariales o institucionales).

- De integración social.

En el caso que nos ocupa la transformación de la fundación no ha sido en cooperativa de integración social, sino en cooperativa de trabajo asociado sin ánimo de lucro y de iniciativa social que tiene por finalidad la integración social. Y cooperativas de trabajo asociado ${ }^{4}$ son «aquellas que tienen por objeto mantener o mejorar para los socios puestos de trabajo a tiempo parcial o completo mediante la organización en común de la producción de bienes o servicios para terceros y, en general, el poder de autoorganización y gestión democrática de la sociedad, sea cual fuere la duración, periodicidad, intensidad o continuidad de dichos esfuerzos y el sector económico en que los mismos se desarrollen».

f) Clases de socios en las cooperativas

a) Socios de trabajo

Es el socio trabajador de la cooperativa de segundo o ulterior grado o de la de primer grado que no sea de trabajo asociado o de explotación comunitaria y su actividad consiste en la prestación de su trabajo en la cooperativa (art. 21 LCE).

\section{b) SOCIO TRABAJADOR}

Es el socio de la cooperativa de trabajo asociado. Su actividad consiste en la aportación de trabajo o actividad profesional a la cooperativa de forma continuada. La cooperativa es en estos casos el instrumento que utilizan los socios para organizar su trabajo en común y para que les represente ante terceros.

La aportación de su actividad por parte del socio trabajador es continuada, pero puede ser de duración determinada o indeterminada. Y en el primer caso, con opción a ser contratados de forma indefinida o no (vid. Estatutos de «PEÑASCAL, S.COOP.», art. 5.Cuatro).

\footnotetext{
4 Fernando San José Martínez, Manual de Derecho..., ibid., pp. 103 y ss.
} 


\section{C) SOCIO COLABORADOR}

El que sin participar en la actividad propia de la cooperativa contribuye a su realización. En algunos casos su régimen de colaboración puede establecerse con mayor o menor precisión en los estatutos sociales, que suelen dejar en manos del Consejo Rector la admisión de socios colaboradores con sumisión a lo dispuesto en los propios estatutos y a las decisiones de la Asamblea General.

Según los estatutos de "PEÑASCAL, S.COOP.", las personas físicas o jurídicas que sin poder realizar plenamente el objeto social cooperativo puedan colaborar en la consecución del mismo, ya sea en el ámbito directamente social o cooperativo, ya sea en un ámbito técnico, comercial, financiero o cualquier otro de índole empresarial».

\section{d) Socio InActivo}

Con diversas denominaciones (socio inactivo, socio excedente, socio honorífico, etc.), se admiten en la legislación sobre cooperativas socios que tras haber dejado de realizar la actividad que constituye el objeto de la cooperativa son autorizados a mantener la cualidad de socios, aunque se exigen para ello ciertos requisitos, cuya regulación se suele deferir a los estatutos sociales, que suelen establecer exigencias de previa vinculación a la cooperativa durante un plazo determinado, limitaciones de derechos políticos o económicos, de obligaciones económicas, etc.

\section{e) AsOCIADOS}

Son socios que participan en el capital de la cooperativa, pero no en la actividad propia de la cooperativa. Es una situación que conlleva siempre la limitación de derechos políticos y económicos en la medida que se prevea en los estatutos sociales.

\section{g) La baja de los socios en las cooperativas y el derecho de retorno}

Por ser el principio de adhesión voluntaria y abierta característico de la sociedad cooperativa, los socios pueden darse de baja en cualquier momento y los estatutos deberán regular el derecho de los socios al reembolso de sus aportaciones. Dicho reembolso, salvo en el caso de participaciones especiales, estará sujeto al principio de libre pacto (artículo 64.5 LCE), pudiendo establecerse deducciones tan sólo sobre las 
aportaciones obligatorias, a cuyo fin distingue la LCE en su artículo 63 entre la baja voluntaria y la expulsión en la siguiente forma:

a) En caso de baja voluntaria y no justificada, las deducciones podrán alcanzar hasta el veinte por ciento de dichas aportaciones.

b) En caso de expulsión por alguna de las causas previstas en los estatutos, las deducciones podrán alcanzar hasta el treinta por ciento.

c) En caso de que se hubiese pactado un período de permanencia mínimo y el socio pidiese la baja antes de que dicho período haya concluido, los porcentajes de deducción pueden incrementarse hasta en un diez por ciento, en la forma que se prevea en los estatutos sociales.

La decisión acerca del porcentaje de deducción corresponderá siempre a los administradores. Y hay que tener en cuenta que a efectos de determinar dichas deducciones se tendrán en cuenta las pérdidas que consten en el balance de cierre del ejercicio en que se produzca la baja, tanto si corresponden a dicho ejercicio o a otros anteriores, siempre que no hayan sido compensadas.

El plazo máximo de reembolso no podrá exceder de cinco años a partir de la baja, salvo en el caso de fallecimiento, en el que dicho plazo no podrá exceder de un año.

Las cantidades pendientes de reembolso no serán actualizables, pero devengarán intereses al tipo legal.

Además de este derecho al reembolso de aportaciones en caso de baja voluntaria o expulsión, tiene el socio el de retorno cooperativo, que equivale a lo que en las sociedades mercantiles es el dividendo o sea, el derecho de participar en el excedente cooperativo en proporción a su participación en el capital. Pero en esta cuestión hay que distinguir el caso de que la cooperativa tenga ánimo de lucro, en cuyo caso el excedente se distribuye a los socios en proporción a su participación del caso de que la cooperativa, como sucede en "PEÑASCAL, S.COOP.», no tenga ánimo de lucro, en cuyo caso el excedente se reinvierte en la entidad o se destina a obra social.

Por último, hay que mencionar, al hablar del derecho de reembolso, las participaciones especiales que regula el artículo 64 LCE en las cooperativas de crédito y de seguros, que son aquellas financiaciones subordinadas en las que los suscriptores habrán de ser necesariamente entidades no cooperativas, en las cuales el reembolso no podrá tener lugar hasta que transcurran cinco años desde la fecha de emisión y la remuneración se establezca en función de los resultados de la cooperativa. 
La emisión o contratación de dichas participaciones especiales habrá de ser ofrecida en cuantía no inferior al 50\% a los socios y trabajadores asalariados de la cooperativa antes de ofrecerse a terceros.

h) Menciones a la transformación hechas en la escritura de transformación de «Fundación Peñascal» en sociedad cooperativa

La fundación, diciendo que proviene de la unión de dos asociaciones cuyo objeto era dar respuesta a un problema de exclusión social existente en un contexto determinado. Dichas asociaciones habían sido creadas gracias a la voluntad de los promotores de «estar atentos a situaciones potenciales de marginalidad en grupos de alto riesgo». Se partía de actitudes y actuaciones como el voluntariado, la solidaridad y el compromiso por atender a situaciones de exclusión en un barrio próximo.

Con el transcurso del tiempo se experimentó la necesidad de una forma jurídica profesional que diese más estabilidad a las personas implicadas en el proyecto (o sea, una necesidad interna y de carácter económico en gran parte). Pero la estructura fundacional tuvo, a juicio de los propios partícipes en el proyecto, las siguientes limitaciones:

a) El Patronato, que tiene como misión velar por el cumplimiento de los fines de la entidad y controlar la buena marcha del proyecto no podía delegar ni compartir su responsabilidad con otro órgano de la fundación que posibilitase un modelo de cogestión o gestión compartida.

b) La participación de los trabajadores se limitaba al cumplimiento de su trabajo y a sentir vocación para el mismo, sin que su participación en las decisiones de futuro fuese obligatoria, «hecho que cada día es más determinante en proyectos sociales para que una organización pueda mantenerse en el tiempo».

c) La voluntad colectiva es la de los patronos. Como mucho, los trabajadores serán consultados, pero sus aportaciones no tendrán carácter decisorio, pues dicha responsabilidad recae por ley en los patronos (esto, supongo, en una organización como la que nos ocupa, no obstaría mucho a que las «aportaciones» de los trabajadores fueran tenidas en cuenta).

d) «... Inmersos en una crisis que está afectando a todos los sectores, incluida la Administración, se hace más inminente la necesidad de buscar estructuras que posibiliten la flexibilización de las relaciones laborales como clave para hacer viables los proyectos 
sociales, que cada día deben atender a más personas afectadas por la denominada crisis con un número de recursos cada vez menores. La contribución de los profesionales en lo económico de la organización se hace cada día más imprescindible.»

e) Como consecuencia de la transformación, la implicación de los socios en el proyecto colectivo será mayor, se dará la solidaridad retributiva entre los socios con un modelo de reparo equilibrado y basado en el esfuerzo y en los logros obtenidos. El patronato entiende que se conservarán en todo momento la personalidad jurídica y las señas de identidad del proyecto y que la apuesta colectiva seguirá siendo la de dar respuesta a situaciones de exclusión y marginalidad.

\section{i) Compromiso}

Como condición indispensable para la transformación, la fundación constituirá un fondo de reserva de transformación irrepartible equivalente al valor patrimonial de la propia fundación. Y el control de dicho fondo corresponderá al CSCE, así como la ampliación o modificación del objeto social de la cooperativa. Una cooperativa que, recordémoslo, carece de ánimo de lucro. Con ello se pretende garantizar que el patrimonio, a través de ese fondo de reserva se siga destinando exclusivamente a los mismos fines que tenía en la fundación. Y para ello, el CSCE, en sesión ordinaria de su Comisión de Régimen Jurídico de 13 de diciembre de 2011, acordó aceptar el control y tutelaje del citado fondo y velar para que se destine a los exclusivos fines de la fundación, reservándose la facultad de autorizar las variaciones en el mismo.

En este caso se produce una especie de subsistencia de la figura de la fundación como patrimonio afecto a un fin, lo que da lugar a una entidad un tanto mixta, a la existencia de una cooperativa con una especie de "sección fundacional» (a tal efecto, cabe recordar que el artículo 6.1 de la LCE admite la existencia de secciones capaces de adoptar acuerdos propios, si bien dispone que deberán llevar contabilidad independiente. Cabe preguntarse qué ocurrirá si ese fondo irrepartible no se distribuye entre los socios pero se agota mediante su empleo en la finalidad de la fundación. Y cabe preguntarse también si lo que se ha constituido es una cooperativa que tiene por objeto gestionar el patrimonio que perteneció a una fundación, pues lo que parece claro es que, si se produce una sucesión a título universal en el patrimonio de la fundación por parte de la Cooperativa, ésta podrá disponer de dicho patrimonio para los fines sociales, aunque, eso sí, sin repartirlo entre 
los socios, con la limitación, prevista en la disposición final cuarta, de que el CSCE controlará el «blindaje» de dicho fondo, siendo necesaria su autorización para las posibles variaciones que la Cooperativa, en cualquier momento, «pudiera solicitar, aun cuando fuesen aprobadas por su Asamblea».

Sin embargo, según dispone la LCE en el artículo 1.2, «la cooperativa deberá ajustar su estructura y funcionamiento a los principios cooperativos, que serán aplicados en el marco de la Ley. Dentro de ésta, actuará con plena autonomía e independencia respecto de cualesquiera organizaciones y entidades, públicas o privadas». Cabe dudar si este mandato legal ha sido plenamente respetado, en el delicado encaje de la transformación, por los estatutos de la cooperativa, aunque así debe de haberlo entendido el Registro, que ha considerado inscribible el acuerdo. Y una vez inscrita la cooperativa, inscripción que tendrá carácter constitutivo (art. 16.2 LCE), la acción de nulidad sólo podrá ejercitarse por las causas y con los efectos establecidos en la LSA (art. 14 LCE).

Por eso el CSCE, en sesión ordinaria de la Comisión de Régimen Jurídico de 13-10-2011, adopta por unanimidad en el caso de la SOCIEDAD COOPERATIVA PEÑASCAL, S.COOP. El siguiente acuerdo: "Aceptar el control y tutelaje del Fondo de Reserva de Transformación Irrepartible existente en la SOCIEDAD COOPERATIVA PEÑASCAL, S.COOP., correspondiente al valor patrimonial de fundación, de la entidad Fundación Peñascal y que, como consecuencia de la transformación de la misma en Cooperativa se ha constituido como Fondo Irrepartible.

El CSCE, de conformidad con la voluntad de la Fundación transformada, velará para que los fondos consignados en el citado Fondo se destinen a los exclusivos fines para los que fueron destinados en la Fundación Peñascal.

Para ello, el CSCE deberá autorizar las posibles variaciones que la Cooperativa pueda realizar en el mismo (en el Fondo), aun cuando deban ser aprobadas por la Asamblea de la misma. Asimismo, el CSCE, al objeto de garantizar el citado fondo, deberá autorizar la ampliación o modificación del objeto social de la Cooperativa, si así fuera acordado por la Asamblea de la Cooperativa».

\section{j) La cooperativa de integración social}

Sin olvidar que «PEÑASCAL, S.COOP.» es una cooperativa de trabajo asociado que tiene como fin la integración social, y no una coo- 
perativa de integración social, dejo constancia en esta intervención mía de la existencia de esta clase de cooperativas, que tienen como finalidad la integración social y la inserción laboral de minusválidos y desfavorecidos y que deben estar formadas mayoritariamente por socios que tengan reconocida administrativamente su minusvalía física o psíquica.

Están reguladas por la LCE en la sección $\mathrm{XI}$, que consta de un solo artículo, el 127, que dice así:

\section{Artículo 127. Sujetos y modalidades}

1. Las cooperativas de integración social estarán constituidas, al menos mayoritariamente, por disminuidos físicos o psíquicos, y podrán basarse en el trabajo asociado para organizar, canalizar y comercializar los productos y servicios del trabajo de los socios o ir dirigidas a facilitar la provisión de bienes y servicios de consumo general o específicos.

2. En las cooperativas de integración social podrán participar como socios entidades públicas responsables de la prestación de cualesquiera servicios sociales mediante la correspondiente aportación y la designación de un representante de las entidades públicas. Este representante prestará su trabajo personal de asistencia técnica, profesional y social junto a los socios de la cooperativa y asistirá con voz a las reuniones de todos los órganos sociales.

Y tienen como finalidad única la integración social de personas que se encuentren en esa situación, de manera que si el número de socios de esa clase dejara de ser mayoritario la cooperativa debe disolverse o transformarse en cooperativa de otra clase.

\section{k) La transformación de fundación en cooperativa desde una perspectiva fiscal}

Desde el punto de vista fiscal, considero suficiente señalar que la operación objeto de estas notas es en realidad la constitución, por transformación de otra clase de entidad, de una sociedad cooperativa, sujeta al tipo impositivo que grava las operaciones societarias dentro de la Norma Foral 1/2011, de 24 de marzo, del Impuesto de Transmisiones Patrimoniales y Actos Jurídicos Documentados (art. 6.B.1, 2 y 3), con arreglo al tipo impositivo correspondiente conforme a los artículos 31 y 38 de dicha Norma Foral, que es del 1\% sobre la base del capital social, si bien en este caso resulta aplicable la exención prevista en el artículo 57.2 de la citada Norma Foral. 
I) La sociedad cooperativa pequeña de la Ley 6/2008

Como una especialidad dentro de las sociedades cooperativas en Euskadi, es necesario mencionar la sociedad cooperativa pequeña, que puede definirse como aquella sociedad cooperativa de primer grado perteneciente a la categoría de cooperativas de trabajo asociado o de explotación comunitaria que se rige por la Ley 6/2008, de 25 de junio.

Esta clase cooperativas ha de constituirse expresamente con el carácter y denominación de sociedad cooperativa pequeña sometida a la Ley que específicamente las regula, ha de tener un número mínimo de dos socios y máximo de diez y se ha de constituir en escritura pública, adquiriendo su personalidad jurídica por medio de su inscripción en el libro especial de sociedades cooperativas pequeñas del Registro de Cooperativas de Euskadi.

La escritura de constitución deberá contener los mismos requisitos que para la constitución de las Cooperativas exige el artículo 12 de la Ley 4/1993, si bien el contenido de los estatutos sociales ha de ajustarse a un modelo orientativo establecido en la Ley 6/2008, de 25 de junio, de la Sociedad Cooperativa Pequeña de Euskadi 5 .

Por lo demás, no parece que las sociedades cooperativas pequeñas puedan surgir como consecuencia de la transformación de una fundación, toda vez que no hay en la Ley que las regula una disposición semejante al artículo 86 LCE y que el artículo 1.2 de la Ley de Cooperativas Pequeñas del País Vasco habla tan sólo de «transformación de una sociedad no cooperativa en cooperativa», sin aludir a la transformación en cooperativas pequeñas de otra clase de entidades, lo que cierra el paso a la transformación directa de una fundación en sociedad cooperativa pequeña.

\section{Notas bibliográficas}

GaRRIGUes, Joaquín: Curso de Derecho Mercantil, I.

Ginto Monzón, Carlos Martín: Manual de Derecho de Sociedades Cooperativas, Santiago MerINo (coord.), CSCE y GEZKI, Vitoria-Gasteiz, 2007.

San José Martínez, Fernando; Manual de Derecho de Sociedades Cooperativas,

Santiago MerINo (coord.), CSCE y GEZKI, Vitoria-Gasteiz, 2007.

UríA, Rodrigo: Derecho Mercantil, Marcial Pons. Madrid, 1989.

5 Publicado en el Boletín Oficial del País Vasco, número 127, de 4 de julio de 2008. 